Archives

6 | 1990

Varia

\title{
La corporation des peintres et la censure des images à Bologne au temps des Carrache
}

\section{Roberto Zapperi}

\section{(2) OpenEdition}

Journals

Édition électronique

URL : http://journals.openedition.org/ccrh/2872

DOI : $10.4000 /$ ccrh.2872

ISSN : $1760-7906$

\section{Éditeur}

Centre de recherches historiques - EHESS

Édition imprimée

Date de publication : 15 octobre 1990

ISSN : 0990-9141

Référence électronique

Roberto Zapperi, «La corporation des peintres et la censure des images à Bologne au temps des Carrache ", Les Cahiers du Centre de Recherches Historiques [En ligne], 6| 1990, mis en ligne le 20 mars 2009, consulté le 20 avril 2019. URL : http://journals.openedition.org/ccrh/2872 ; DOI : 10.4000/ ccrh.2872

Ce document a été généré automatiquement le 20 avril 2019.

Article L.111-1 du Code de la propriété intellectuelle. 


\title{
La corporation des peintres et la censure des images à Bologne au temps des Carrache
}

\author{
Roberto Zapperi
}

1 Dans l'Italie du XVI ${ }^{e}$ siècle, pour travailler et ouvrir boutique en ville, les artisans devaient être en possession d'une patente professionnelle et faire partie de la corporation correspondant à leur art. L'accès à cette corporation était strictement réglementé. Ceux qui ne remplissaient pas toutes les conditions requises et qui ne pouvaient faire la preuve, en particulier, que leur famille résidait dans la ville depuis trois générations, devaient se résigner à travailler comme subalternes, comme « dépendants ». Cette dénomination dit clairement leur situation à l'égard de la corporation qui exerçait sur eux son contrôle et leur imposait un lourd tribut. Il est évident que ces exigences reposaient sur la crainte de la concurrence, propre à l'économie traditionnelle, et sur le désir qu'avait la corporation d'exclure les étrangers de la ville.

Les peintres n'échappaient pas à cette règle ; comme travailleurs manuels ils entraient dans la catégorie des artisans et pour pouvoir exercer leur métier il leur fallait donc s'agréger à quelque corporation. Comme ils atteignaient en effet difficilement le nombre de maîtres voulu par les règlements en vigueur, ils ne pouvaient donc que rarement constituer une corporation qui leur fût propre. À Bologne ils sont restés au-dessous du chiffre minimum pendant des siècles et ont dû s'intégrer à la corporation dite des « quatre arts » qui regroupait les selliers et les fabricants d'épée et de fourreaux. En plein $\mathrm{XVI}^{\mathrm{e}}$ siècle, à un moment où la peinture jouissait d'un grand prestige, les maitres les plus riches et les plus célèbres se sentaient humiliés de cette promiscuité. Des artistes comme Prospero Fontana, Bartolomeo Passarotti, Lorenzo Sabatini et Orazio Samacchini avaient fait fortune; ils avaient travaillé hors de Bologne, à Florence et à Rome, parfois même pour des papes ou des cardinaux. Ils ne supportaient plus d'être confondus avec des artisans de si bas niveau. En 1569, ils réussirent à s'en séparer en créant une nouvelle corporation dans laquelle ils durent pourtant admettre des bombasari ou travailleurs $\mathrm{du}$ coton, du bombasin plus précisément, pour atteindre le nombre de maîtres 
réglementaire. Les bombasari étaient encore moins nombreux que les peintres et, comme ils ne cessaient de diminuer, ils ne comptaient pratiquement plus pour rien.

Conformément aux statuts approuvés par le Sénat, la corporation devait être représentée et gouvernée par un conseil composé de vingt peintres et de dix bombasari et il fallut admettre, pour faire nombre, quelques artisans d'une autre profession, comme le parfumeur ou droguiste Giacomo Pennacchini. Le conseil à son tour, élisait un massaro pour trois mois, deux consuls et un syndic pour six mois. Ces quatre maitres, avec à leur tête le massaro, constituaient l'exécutif, ils étaient chargés de faire valoir l'autorité de la corporation sur tous les artisans qui exerçaient des métiers relevant de sa compétence: peintres et bombasari bien sûr mais aussi tous les artisans qui usaient pour leur métier du bombasin, des pinceaux ou des couleurs. Le plus clair de leur charge consistait à percevoir les contributions et à éliminer toute forme de concurrence. Les statuts interdisaient explicitement, sous peine d'une très forte amende, à tous les artisans, maîtres ou «dépendants » de prendre la succession d'un collègue dans un travail sans avoir obtenu au préalable l'accord de la corporation ou l'autorisation du conseil ${ }^{1}$.

4 Les «dépendants" et les maîtres étaient tenus de payer, pour les ouvriers qui travaillaient dans leur boutique, des contributions dont le recouvrement était particulièrement ardu. Les archives de la corporation des peintres ont en grande partie disparu et il ne reste aucune des listes où devaient être inscrits les « dépendants » et les ouvriers. On sait pourtant que de nombreux artisans - et, parmi eux, un bon nombre de peintres - travaillaient et tenaient même boutique sans payer aucune taxe ni se faire jamais inscrire. C'est ce dont témoignent les avis que publiaient de temps en temps, sur requête des massari, les autorités de la ville, le légat ou le vice-légat. On y menaçait de lourdes amendes les «dépendants» qui ne s'inscriraient pas et les maîtres qui ne déclareraient pas leurs ouvriers et ne paieraient pas leurs taxes. Mais les avis restaient sans effet. Aussi le conseil, pour maintenir son autorité sur tous les peintres qui exerçaient à Bologne, et afin d'encaisser les sommes qui lui étaient dues, recourait-il périodiquement à la vente aux enchères des impôts, prévue implicitement par les textes de 1569. Il en cédait le recouvrement à un adjudicataire qui savait généralement fort bien comment s'y prendre pour obtenir ce qu'il voulait. Procédures hâtives, brimades, exactions: ces vexations inévitables suscitaient des protestations et des controverses juridiques interminables, pour lesquelles on recourait souvent aux plus hautes autorités.

En 1586 éclate un de ces litiges. Il traîne toute l'année suivante et les autorités finissent par intervenir. Une augmentation illégale du montant des contributions avait déclenché la colère des «dépendants » les plus pauvres. En juin 1586, la révolte est à son comble : l'un des "dépendants ", le peintre Giovanni Ferrarese, un étranger précisément, fait irruption dans le conseil escorté d'une foule de compagnons en colère. La bagarre est évitée de justesse. Dans le procès qui suit cet incident, les deux conseillers Pospero Fontana et Giovanni Battista Ramenghi viennent témoigner en faveur du consul Felice Pinarizzi. Dans un mémoire adressé au cardinal Enrico Caetani le 19 octobre 1587, les « dépendants » se déclarèrent prêts à payer la somme habituelle dont ils sont redevables dans la mesure où le leur permet leur pauvreté. Ce rappel de leur pauvreté produit l'effet voulu. Le légat intervient et, en bon Salomon, décide de laisser les choses en l'état: les "dépendants » devront payer certes, mais la somme habituelle, sans augmentation. La nature sociale du conflit ne fait aucun doute: les maîtres les plus côtés cherchaient à exclure de la corporation les peintres étrangers et les plus pauvres. Ceux-là, faute de moyens artistiques et économiques, allaient se joindre aux artisans qui exerçaient des 
métiers voisins des leurs. Exclusion donc, mais aussi exploitation, car les maîtres les soumettaient à une taxe semestrielle de vingt sous qui servait à financer les frais de la corporation et à payer de somptueux salaires aux maitres qui se relayaient dans les tâches de direction ${ }^{2}$.

Les incidents de 1586-87 mettent en lumière le conflit social mais aussi les rapports de pouvoir qui se jouaient au sein même de la corporation, et leur lien étroit avec le problème crucial de la concurrence. Dans notre documentation apparaissent les noms de quatre peintres ; les deux premiers, Fontana et Ramenghi, figurent parmi les maitres les plus célèbres et les plus prestigieux, les deux autres sont inconnus et se situaient probablement tout en bas de l'échelle professionnelle. L'un d'eux, Giovanni Ferrarese, était un étranger, simple "dépendant ", mais l'autre, Felice Pinarizzi, très certainement de Bologne, avait été admis dans la corporation comme maître, coopté dans le conseil et élu consul. Pinarizzi ne fait pas partie, comme Fontana et Ramenghi, des membres qui ont fondé la corporation en 1569 ; il y a été admis plus tard, comme maitre, et a été coopté dans le conseil en 1577, en même temps qu'un obscur artisan, un certain Angleo Desegna, par un vote que le Sénat a sanctionné le 23 août. Il doit son entrée dans le conseil à la mort, au début de juin, d'un autre maître éminent, Orazio Samacchini. Le Conseil se réunit le 15 juillet pour pourvoir le poste resté vacant, mais aucun des peintres qui présentaient leur candidature n'obtint le nombre de voix suffisant. Pour Malvasia, qui a écrit au XVII ${ }^{\mathrm{e}}$ siècle la vie des peintres de Bologne, les procès-verbaux des séances indiquent que le conseil aurait pensé « offenser la mémoire honorée et vertueuse » du défunt en lui donnant pour successeur un de ces autres maitres qui lui étaient tous bien inférieurs.

7 En réalité l'un de ces quatre peintres, Cesare Baglione, qu'ailleurs Malvasia qualifie d'«esprit vif, plein de talent et de force ", était sans conteste très supérieur aux trois autres et l'accord aurait dû se faire sur son nom. Mais, dit Malvasia, avec le temps l'activité de Baglione «ne correspondit plus à ce qu'on attendait de lui ». Déception qu'apparemment le Conseil partageait, puisqu'au cours de la deuxième séance il préféra à Baglione deux de ses trois concurrents, Desegna et Pinarizzi, dont on n'attendait strictement rien. Dans la première séance, Baglione avait été très injustement classé au même rang que trois autres peintres, et dans la deuxième il fut carrément exclu au profit de ceux de ces trois nullités. Desegna prit la place de Samacchini. Il ne restait à Baglione, qui ne manquait pas d'esprit, qu'à se consoler de ce choix extravagant par un jeu de mots. «Les gens du Conseil, dit-il, se contentent aujourd'hui de celui qui peine sur son dessin, sans se soucier qu'il peigne bien ». On comprend les sarcasmes de Baglione, qui était à l'époque un peintre confirmé. L'un des conseillers les plus influents, Fontana, le savait bien. Il l'avait eu en effet comme collaborateur en maintes occasions, à Florence, à Parme, à Città di Castello; il appréciait chez lui le fini de sa peinture et les spécialités qu'il avait choisies comme le paysage, le dessin ornemental, les grotesques. Par ailleurs, depuis 1574, Baglione recevait un traitement des Farnèse. Or travailler pour une cour représentait à cette époque un titre de mérite exceptionnellement important. Aussi faut-il comprendre son exclusion du Conseil où Pinarrizi fut admis par la suite pour pourvoir une seconde place restée vacante, comme une mesure destinée à barrer la route à un concurrent dangereux. Le souci de tenir sous contrôle les concurrents de valeur explique l'extrême prudence avec laquelle le Conseil procédait au remplacement des membres après leur mort. S'il ne trouvait pas parmi les maîtres disponibles des gens sûrs et prêts à s'incliner bassement devant la volonté des notables, il préférait laisser la place vacante ${ }^{3}$. 
8 Une liste de conseillers en date du 1er octobre 1581 atteste effectivement qu'après avoir été vingt à l'origine, ils n'étaient plus que quinze. Un décret du Sénat du 31 janvier 1584 légalise même cette réduction forcée des membres. De toutes façons la liste parle d'elle même : sur les quinze peintres qui y figurent, dix sont de pauvres tâcherons dont il est impossible de retrouver la moindre trace, la moindre oeuvre, la mention même du moindre tableau. Sur quelques-uns d'entre eux, comme Paolo Zagnoni (" peintre très ordinaire ») ou Giuliano Spinelli (qui fait « de la peinture et de la dorure » mais « ne vaut pas grand chose ni dans l'une ni dans l'autre »), Malvasia a réussi à recueillir quelques informations, d'ailleurs peu flatteuses. Ils étaient au Conseil pour faire montre et permettre à la corporation de subsister. Pour le reste, ils étaient dans la dépendance des cinq autres membres, qui assuraient la direction effective de la corporation même quand ils n'en avaient pas la charge officielle. Il s'agissait des représentants les plus connus de la peinture bolognaise de l'époque, Prospero Fontana, Ercole Procaccini, Giovanni Battista Ramenghi et Domenico Tibaldi. Fontana, qui était le plus important d'entre eux faisait selon Malvasia la pluie et le beau temps; il se mêlait de tout, faisait l'arbitre entre les peintres ou même entre les peintres et les clients : une sorte d'oracle, en somme, auquel personne n'osait s'opposer.

9 Fontana, durant cette période, avait été converti par le cardinal de Bologne, Gabriele Paleotti, à la nouvelle religion de la peinture de la Contre-Réforme. C'en était désormais fini des recherches formelles du maniérisme et de ses audaces d'expression: seul comptait le contenu, c'est à dire la transmission d'un message qui visait à l'édification des fidèles et auquel le nihil obstat des autorités ecclésiastiques garantissait une orthodoxie parfaite. Avec cette conversion c'était la vieille génération maniériste de Bologne qui se rendait. Fontana ne s'est pas contenté en effet de collaborer au Discours sur les images sacrées et profanes publié en 1582, par lequel le Cardinal fixait les nouvelles règles de la peinture sacrée ; il a mis tout en oeuvre pour entraîner à sa suite collègues et élèves et les amener à la pratique de la peinture dévote. Et il est difficile de ne pas le soupçonner d'avoir usé du pouvoir que sa position lui donnait sur la corporation pour arriver à ses fins. Il suffit d'ailleurs, pour s'en convaincre, de considérer les quelques artistes un peu prestigieux de la corporation, tous aussi dévoués que lui à Paleotti. D'autre part, sur les cinq peintres qui avaient une certaine importance, quatre étaient associés, directement ou indirectement, à la réalisation du projet de l'évêque qui devait assurer dans les années 1580, comme le dit Daniele Benati, «le modèle indispensable pour la peinture de la Contre-Réforme à Bologne ». C'est ce que prouve la peinture des fresques de la crypte du dôme que l'architecte de l'évêché, Tibaldi, avait aménagée pour les recevoir : ont en effet participé à leur réalisation Fontana lui même, qui a fait l'une des premières en 1579 et qui a peut-être supervisé les autres, mais aussi Giovanni Battista Fiorini et le jeune Camillo Procaccini, que son père a bien évidemment mis à sa propre place. Quant au cinquième conseiller, Giovanni Battista Ramenghi, s'il n'a pas participé à ces fresques, ses oeuvres de l'époque montrent qu'il se conformait strictement aux directives de Paleotti, encouragé en cela par Fontana qui avait été son maître ${ }^{4}$.

10 Le rôle décisif qu'a joué Paleotti dans l'évolution des arts visuels à Bologne ressort déjà de la nature du Discours, ensemble de conseils pratiques et de prescriptions qui s'avoue dès le début comme un instrument d'action pastorale. Et même s'il n'a été tiré qu'à un petit nombre d'exemplaires réservés à des lecteurs choisis par l'auteur, il est sûr que Fontana en possédait un. Quoiqu'il en soit le Discours n'est pas dû au hasard, il est le résultat d'une vaste expérience pastorale. Paleotti, en effet, prit la décision de l'écrire après avoir 
inspecté personnellement (ou fait inspecter par des ecclésiastiques de confiance) les édifices sacrés du diocèse. Il avait alors constaté que les peintures qui y étaient exposées n'étaient pas conformes aux normes établies par le concile de Trente, à l'élaboration desquelles il avait d'ailleurs lui-même participé. Ses visites pastorales n'ont jamais été étudiées systématiquement de ce point de vue, mais un sondage, portant sur un certain nombre de visites qu'il rendit personnellement en 1576 dans les Apennins et en 1580 à Bologne même permet d'arriver à cette conclusion.

11 On voit en effet dans les actes de ces visites que l'évêque a donné l'ordre, sous une forme souvent péremptoire, de « remettre à neuf » les peintures. Il ne fournit généralement pas la moindre justification. La note ordonnant «qu'on remette à neuf » ou "qu'on nettoie les figures de la chapelle» (Bologne, église Santa Maria Maggiore) concerne clairement la restauration d'un tableau vieux et sale. Mais cette autre note: « restaurer le retable de l'autel avec de nouvelles peintures dans lesquelles on introduira la figure de saint Christophe » est, de son côté, sans équivoque. Il s'agit là de refaire le tableau; et comme à Sassuno, dans l'Apennin bolonais, l'église était dédiée à ce saint, on demandait qu'il y fût représenté en précisant toutefois que "saint Christophe vient après saint Pierre ». L'évêque a certainement trouvé à redire aussi aux peintres de l'abside puisqu'il a donné l'ordre de «remettre à neuf la tribune et de la décorer de peintures convenables », en précisant à nouveau que "Saint Pierre vient avant Saint Christophe ». C'est là l'unique cas où il justifie ses ordres par des raisons de nature idéologique. Dans l'église de San Michele à Montepiano il s'est contenté de demander que «soient effacées les lettres qui sont sous la figure du Christ dans le tableau d'autel ». Ailleurs il a ordonné laconiquement de «faire un tableau d'autel avec des figures convenables» (Sassonero, église de San Mamolo), de "remettre à neuf toutes les figures ou d'en faire d'autres" (Gorgognano, église de San Lorenzo). "Qu'on peigne un tableau d'autel décent » (Bologne, église de Santa Maria Maggiore), «qu'on peigne dans la chapelle de bonnes figures ou qu'on la blanchisse et qu'on la fasse repeindre ensuite» (Bologne, église San Stefano), «qu'on remette les figures à neuf sur les murs ou bien qu'on fasse un beau tableau d'autel» (ibid.), « qu'on fasse un tableau d'autel décent avec les images du titre » (Bologne, église Santa Maria della Mascarella).

Il est clair que toutes ces retouches, destructions, rénovations et commandes de peintures nouvelles sont dictées, même si l'évêque ne l'avoue jamais, par des impératifs théologiques et moraux. On ne peut s'expliquer autrement que de telles dépenses aient été engagées alors qu'il « était parfaitement possible de procéder à des restaurations peu onéreuses. La primauté de Pierre, la suppression des inscriptions incongrues, la décence, voilà un échantillon des critères que l'on trouve multipliés et développés dans le Discours. On peut lire à travers les notes nécessairement lapidaires des actes des visites que ces invites permanentes à "remettre à neuf» visaient à combattre les abus dont il est amplement question dans le Discours.

Paleotti faisait souvent une seconde visite de contrôle, car ses instructions n'étaient pas toujours respectées. Mais il n'a pas pris tout de suite la voie de la répression. Il s'est contenté au début de s'attaquer aux images lascives : en 1573 il a fait imprimer et afficher des Avertissements aux pères de famille dans lesquels il les invitait instamment à faire disparaître "de la maison toute peinture et tout livre lascifs et indécents et à n'y conserver que des images honnêtes et des représentations de saints, de sorte qu'on sût qu'on était dans la maison d'un chrétien et non dans celle d'un gentil». En 1577, il a recommandé aux prédicateurs de «reprendre ceux qui gardent chez eux des images 
lascives et des livres malhonnêtes et vains ». Il n'a dédié qu'un seul édit en 1575 à la peinture sacrée, pour rappeler aux particuliers qui en exposaient devant leur maison qu'ils devaient restaurer leurs tableaux. Cette absence d'édit et ce silence ne peut-être le signe d'un désintérêt de la part de l'évêque car, en 1578, il avait commencé son Discours et s'occupait de la peinture sacrée dans ses visites pastorales. Mais il avait choisi une autre voie : il cherchait plutôt, par une campagne discrète et diffuse et par l'usage d'arguments judicieux à convaincre les gens et en particulier les peintres, à qui il s'adressait individuellement ou à travers leur corporation. Il savait parfaitement quel contrôle le Conseil de la corporation exerçait sur la peinture, et il le rappelle à plusieurs reprises très clairement dans son Discours. Ce sont en effet, déclare-t-il, «les chefs de l'art et les députés du public qui auront à prendre des mesures » contre les peintres qui se seront rendus responsables d'«œuvres inconvenantes» pour ne pas avoir respecté les "principes et fondements de l'art ». C'est reconnaître clairement que la corporation était la seule compétente pour juger et contrôler l'oeuvre du peintre dans son aspect technique, dans «ce qui relève de l'art du dessin » («que le peintre veille à ce que son oeuvre ne rencontre aucune opposition sur ce plan et qu'elle soit approuvée par les gens de l'art»). Mais la valeur du peintre ne se résume pas à ses qualités techniques, et il devait faire preuve, à côté de son "habileté à représenter », d'un souci de «la fin, de la nature, et de l'usage de ce qui est figuré ». Or en cette matière c'étaient, en dernière instance, les "personnes érudites dans le sujet du tableau » et donc, pour la peinture sacrée, les ecclésiastiques qui étaient chargés d'émettre un jugement. Paleotti ne se contentait pas de maintenir en matière doctrinale le peintre dans la subordination étroite des théologiens, il prétendait encore qu'il suivit son enseignement religieux et qu'il y conformât sa peinture.

14 Il aimait en effet citer «l'exemple d'un peintre fort dévot de (son) temps qui, avant de peindre l'image d'un saint, lisait attentivement sa vie. Puis, après s'être pénétré de ses vertus, il peignait en sorte que la représentation suscitât en lui la conjonction et incitât les autres à la dévotion». Les peintres qui ne se soumettaient pas à cette règle commettaient une «erreur très grave, tout à fait contraire à leur art, et péchaient en particulier dans l'imitation ». Paleotti renvoyait, pour un exposé plus détaillé de ces procédures, au cinquième livre, qu'il n'a d'ailleurs jamais écrit. Dans l'immédiat, il se contentait de recommander aux peintres de soumettre, avant exposition, leurs peintures à l'examen "d'ecclésiastiques et d'hommes de lettres pourvus d'un bon jugement et éloignés de toute adulation", mais certainement aussi de peintres dévots, ceux qu'il appelle les «théologiens muets» et les "prédicateurs silencieux du peuple». L'intervention des collègues qui devaient en principe, conformément aux institutions, se borner à prononcer sur l'aspect technique de l'oeuvre, s'étendait ainsi au contenu. Le peintre se trouvait donc soumis à de très fortes pressions idéologiques 5 .

Quand la corporation s'alignait sur ses positions, l'évêque pouvait, sans avoir recours aux sanctions courantes dans l'Italie de la Contre-Réforme, obtenir des peintres qu'ils se conforment aux prescriptions du Concile de Trente. C'est pourquoi il préférait faire appel à la collaboration des catégories intéressées plutôt que de s'en remettre à l'action du Saint-Office de Bologne, qui ne dépendait pas de son autorité. Personne ne s'est occupé des interventions de l'Inquisition dans le domaine précis de la peinture et pourtant, durant le seul épiscopat de Paleotti, quatre peintres au moins ont été convaincus d'hérésie luthérienne et ont fini sur le bûcher, trois en 1567 et un en 1573. On ne sait pas si ces peintures figuraient parmi les chefs d'accusation. La documentation qui nous est 
parvenue sur l'activité du Saint-office à Bologne à cette date est en effet très pauvre, et il n'a pas été possible de retrouver la trace de procès du genre de celui que l'Inquisition de Venise a intenté en 1573 à Véronèse pour la Dernière Cène que le peintre a dû censurer et transformer en Le repas chez Levi'

Paleotti comptait aussi sur les peintres pour convaincre les commanditaires qui représentaient le dernier anneau de la chaîne et le point faible de son entreprise. Il ne pouvait pas compter, en effet, sur la collaboration de ces gens-là qui échappaient à son emprise à la mesure de leur place dans la société ou de leur pouvoir dans la ville. Et si, dans un cas qui fit grand bruit, il réussit à imposer sa volonté contre un peintre étranger de haut renom, il ne dut ce succès qu'à l'intervention active de la corporation.

Federico Zucarri travaillait pour le compte de Grégoire XIII aux fresques de la chapelle Paolina au Vatican quand l'écuyer tranchant secret du pape, Paolo Ghiselli, un bolonais lui aussi, lui commanda un grand tableau pour la chapelle de l'église Santa Maria del Baraccano de Bologne. Il choisit pour sujet la Procession de saint Grégoire le Grand, qui rappelait comment saint Grégoire avait miraculeusement mis fin à une épidémie de peste. Selon une habitude assez répandue à cette époque, Ghiselli fit peindre le saint pape $\mathrm{du} \mathrm{VI}^{\mathrm{e}}$ siècle sous les traits de son heureux successeur Grégoire XIII. Il tint aussi à apparaître personnellement dans le tableau et à y faire représenter à ses côtés le maître de chambre pontifical, Ludovico Bianchetti, lui aussi originaire de Bologne. On peut les voir, agenouillés derrière le pape, habillés en prélats $\mathrm{du}_{\mathrm{VI}} \mathrm{e}^{\mathrm{e}}$ siècle. Dans son Discours, prêt pour l'impression au printemps de 1581, Paleotti s'était déjà insurgé contre l'habitude de peindre les saints «sous les traits de particuliers et de gens du monde connus aux autres »; c'était, disait-il, « chose vaine et proprement indigne ». Mais ni Ghiselli à Rome, ni ses frères à Bologne ne pouvaient rien savoir, car l'évêque avait réservé jusqu'alors ses réflexions sur la peinture sacrée à un petit cercle d'experts, d'amis intimes et de proches collaborateurs. Zucarri termina son tableau à la fin de l'année, mais avant de le remettre à Ghiselli il en fit faire à Rome une gravure sur cuivre par Aliprando Caprioli, qui porte la date 1581. Quand le tableau fut arrivé à Bologne, on procéda à l'inauguration de la chapelle le 12 mars 1581, jour de la fête de saint Grégoire-le-Grand. Paleotti, qui avait été invité à la bénir, assistait en personne à la cérémonie. On peut imaginer son désappointement quand il vit sur le tableau saint Grégoire, l'un des saints les plus célèbres et historiquement l'un des mieux connus, représenté sous les traits d'un personnage contemporain entouré de deux de ses acolytes ${ }^{7}$.

Ce n'était pourtant pas n'importe qui : il s'agissait du pape bolonais qui, depuis des années, lui donnait du fil à retordre, empiétait progressivement sur son domaine et lui disputait son autorité. Paleotti devait agir avec la plus grande prudence s'il voulait obtenir quelque résultat. On ne peut pas dire avec certitude, faute de documents, comment l'évêque s'y prit pour faire enlever le tableau sans heurter la susceptibilité du pontife. Mais on est sûr qu'après son exposition dans l'église de Bologne, les peintres de la corporation firent pression sur les Ghiselli, et il n'est pas invraisemblable de supposer que ce fut sur intervention de Paleotti.

Quoiqu'il en soit, le tableau fut décroché et renvoyé à Rome à Paolo Ghiselli, qui le rendit lui-même à son auteur; il alléguait un rapport écrit des peintres bolonais déclarant ce tableau de trop mauvaise qualité pour figurer dans une chapelle de ce rang. Zuccari ne voulait pas compromettre ses rapports avec le pape ; il savait quelle influence avait sur lui son écuyer. Aussi se montra-t-il conciliant et proposa-t-il de refaire le tableau. Mais pour Ghiselli il n'en était pas question, il lui rendit le tableau, disant qu'il lui semblait fait par 
«le plus vil de tous les peintres et de tous les hommes qui aient jamais pris un pinceau en main ». L'affront n'était pas tolérable. Le 18 octobre 1581, fête de san Luca, patron des peintres, Zuccari exposa à Rome un tableau allégorique intitulé Porta Virtutis dans lequel il disait vertement leur fait à ses détracteurs de Bologne. Mais il eut l'habileté de ne citer personne et d'éviter que quiconque pût se reconnaître.

Cette précaution, en fait, ne lui servit pas à grand chose. Ghiselli fut mis au courant et réussit à comprendre contre qui étaient dirigées les attaques de Zuccari. Et comme personne ne faisait allusion à la présence du pape dans le tableau, et que tout le monde s'en prenait aux piètres qualités de l'oeuvre, il lui fut facile de convaincre son maitre, qui s'y connaissait en peinture aussi bien que lui, de la mauvaise foi de Zuccari. Ce dernier, lui dit-il, avait eu le front de le représenter dans un mauvais tableau auquel il n'avait pas apporté le soin nécessaire et avait ensuite répondu avec arrogance aux critiques que les peintres de Bologne lui adressaient. Il obtint que Zuccari soit jugé et mis au ban de l'Etat pontifical.

Le texte des critiques adressées au tableau n'a malheureusement pas été conservé. On peut déduire des actes du procès intenté à Rome contre Zuccari qu'il ne portait pas de signature et qu'il se contentait d'exprimer « l'avis universel de tous les connaisseurs de la profession ». Il n'est pas difficile de découvrir derrière cette prudente circonlocution la marque de la corporation des peintres de Bologne, qui s'inquiétaient de la concurrence que leur faisait Zuccari. Son tableau fut en effet remplacé par une oeuvre de Cesare Aretusi, approuvée bien entendu par le Conseil comme l'exigeaient les statuts. Mais il y a plus : Fontana et Fiorini, deux des cinq membres importants du Conseil, collaborèrent avec Aretusi ; Fontana prépara l'« invention ", Fiorini exécuta le dessin et la peinture fut confiée à Aretusi qui travaillait en tandem avec lui depuis longtemps.

Le tableau de Zuccari a malheureusement disparu. Mais il en reste l'estampe qu'il avait fait graver avant d'envoyer le tableau à Bologne, ainsi qu'une seconde estampe certainement postérieure, qui présente des différences notables avec la première. Ces différences attestent que Zuccari avait remis la main au tableau qu'on lui avait rendu, et qu'il avait tenu compte en partie, dans ses remaniements, des critiques des bolonais. Si l'on compare les deux estampes avec le tableau d'Aretusi, on voit clairement que derrière les peintres de Bologne se cachait Paleotti. Personne n'a eu le courage de supprimer le visage du pape, mais les peintres bolonais l'ont repoussé encore plus haut dans le tableau et en ont plus ou moins masqué les traits par le jeu des mains qui s'élèvent dans un geste d'imploration. Quant aux visages des deux acolytes, ils ont disparu de la seconde estampe et du tableau des bolonais. Cette disparition vaut la peine qu'on s'y arrête.

23 Les bolonais ont opéré sur ce point une censure beaucoup plus radicale que celle de Zuccari lui-même, qui s'est contenté de changer les traits et de supprimer la mitre des deux acolytes agenouillés derrière le pape. Ils disparaissent au contraire tous deux complètement du tableau d'Aresuti; on ne retrouve même pas leurs visages dans le groupe des spectateurs qui regardent passer la procession. Pour les Ghiselli c'était un affront de taille. Mais Fontana a dû les convaincre que la sainteté du pape, toute liée à sa fonction qu'elle fût, lui conférait des privilèges particuliers et exclusifs.

24 Les peintres de Bologne reprochaient à Zuccari de ne pas avoir respecté les proportions ( « les figures qui se trouvaient devant et qui auraient dû être plus grandes, en raison de la perspective, étaient plus petites et faisaient un effet désastreux»). Cette accusation s'éclaire si l'on remarque que les cadavres des pestiférés que le peintre avait disposés, bien en vue, au premier plan, se trouvent relégués par les peintres bolonais, assez 
indistinctement et avec des proportions réduites, sur le fond du tableau. Au premier plan, débarrassé de ses cadavres, prend place le pape entouré des évêques et des cardinaux. C'est très précisément ce que prescrivait Paleotti dans un chapitre de son Discours consacré aux peintures disproportionnées. Il faisait la critique du peintre qui «n'attribue pas aux choses qu'il figure une place qui corresponde à leur condition et dignité, et qui met sur les côtés ce qui devrait se trouver au centre, ou bien, en oubliant quel est le but principal de l'histoire, il accorde plus de soin, à ce qui a moins d'importance, et attire làdessus le regard ».

Après sa condamnation, le 27 novembre 1581, Zuccari ne se tint pas pour vaincu; il retoucha son tableau - mais il ne changea rien aux cadavres, les laissant au premier plan - et en fit don aux jésuites à condition qu'ils l'exposent dans leur église de Bologne. Ceux-ci l'acceptèrent mais, pour en finir avec toutes les polémiques, ils y firent apposer une inscription en latin, qui fait remonter à la date unique de 1580 la composition et le don de l'oeuvre. Il s'agissait d'annuler par là le laps de temps entre don et composition et, du même coup, d'effacer l'intervention de la censure.

Entre temps Zuccari avait mis des personnages importants sur l'affaire. Avec l'aide des jésuites il réussit à se réconcilier avec le pape: en décembre de 1583 il se remit aux fresques de la chapelle Paolina. Fontana, qui avait espéré récupérer pour lui ce travail, fut déçu. Il faut dire que Ghiselli n'a jamais rien fait pour qu'il l'obtint. En effet, impliqué malgré lui dans l'affaire du tableau, Ghiselli en voulait à Zuccari de l'affront qu'il avait reçu et lui reprochait d'avoir prêté le flanc à la critique bolonaise. Mais il ne portait pas non plus dans son coeur ces peintres de Bologne dont l'intervention ne lui avait pas fait plaisir, au point qu'à sa mort, en 1585 , il ne voulut plus entendre parler de la chapelle de l'église Baraccano et se fit enterrer à San Petronio.

Le grand vainqueur dans cette affaire était Paleotti : il avait réussi à remplacer la toile de Zuccari, il avait obligé le peintre à remanier son tableau, il l'avait soumis à la plupart de ses exigences. L'intervention des peintres de la corporation lui assurait en outre une autre et suprême victoire : il pouvait tranquillement sortir de scène puisque les peintres se chargeaient eux-mêmes d'exercer la censure.

L'influence de l'évêque sur la corporation ne pouvait manquer de susciter des difficultés : on trouve à l'intérieur même du Conseil une victime probable du ralliement général à sa politique. On s'aperçoit en effet, en regardant la liste de 1581, qu'il manque le nom de Passarotti, l'un des peintres les plus éminents de cette époque. Il avait été l'un des leaders des revendications corporatives de 1569, puis avait été massaro au moins deux fois. En principe, la charge de conseiller était une charge à vie. Selon Malvasia, Passarotti a démissionné parce qu'il était en désaccord avec ses collègues sur le problème des taxes que les « dépendants » ne payaient pas. Mais il est difficile de croire que le peintre se soit posé en défenseur des « dépendants ", c'est à dire des artisans les plus pauvres, alors qu'il s'était battu pour se séparer d'eux et qu'il n'avait jamais manqué une occasion de les tourner en ridicule.

29 La vraie raison devait être ailleurs. Le chanoine Malvasia la laisse involontairement transparaitre quand il aborde, aussitôt après, le sujet brûlant du nu dans la peinture sacrée. Passarotti, écrit-il, « fut le premier à Bologne, après le Primatice et Tibaldi, qui a renoncé à l'antique modestie et au respect des maîtres du passé et qui, poussé par l'exemple de Michel-Ange dans son Jugement s'est risqué à introduire des torses nus jusque dans les tableaux d'église ». Il y mit cependant de la modération « comme dans les tableaux, qu'on ne louera jamais assez, qui se trouvent à San Giacomo et dans la chapelle 
de la dogma grossa ». Les deux peintures existent encore et il est facile d'aller contrôler ces dires. Le premier représente la Madonne sur son trône avec l'enfant, entourée de cinq saints. Parmi ceux-ci Jean-Baptiste est représenté demi-nu, au centre, au premier plan. Le tableau se trouve dans l'église de San Giacomo Maggiore et a été daté de 1564-65, années où Paleotti n'était pas encore évêque de Bologne. Le second, la Présentation de Marie au temple, qui se trouve aujourd'hui à la Pinacothèque nationale, date de 1583 ; ce tableau, dont la nudité est bannie, témoigne d'un moment d'extrême prudence dans la peinture sacrée de Passarotti. Ne pas reconnaître la différence entre le premier et le second tableau et juger qu'ils font preuve de la même modération dans le traitement du nu ne peut relever que d'une volonté opportuniste d'amalgame. Car les deux tableaux représentent au contraire deux réponses différentes au même problème, réponses dont la divergence ne s'explique que par l'intervention de Paleotti qui s'est installé à Bologne en 1566. Le saint évêque déplore en effet dans son Discours la fâcheuse habitude qui s'est désormais répandue de peindre les saints, «qui n'ont jamais montré la moindre partie inconvenante de leur corps ", " avec les épaules et les jambes nues, et pire ", exactement comme le Jean-Baptiste de Passarotti. Ce « pire » fort évocateur peut aisément s'appliquer à toute peinture sacrée de Passarotti; il suffit de regarder les nombreuses oeuvres exposées dans les couvents et les églises de la ville et du contado, comme la Résurrection avec la Madonne en prière, Saint Michel Archange, Saint Jean Baptiste, Sainte Lucie, Noli me tangere, Baptême du Christ, Ecce Homo. Le nu n'était certainement pas le seul sujet de friction avec l'évêque. Le Jean Baptiste du tableau de San Giacomo Maggiore est représenté par exemple dans une pose, avec un geste de la main, une expression du visage propres à déclencher l'indignation de Paleotti. L'évêque s'était en effet élevé contre les peintres qui représentent les saints « avec une allure, des gestes et des mouvements plus que licencieux ». L'indécence dépassait évidemment toute limite quand il s'agissait des figures profanes, des personnages de second plan qui n'avaient pas, pour attirer sur eux l'attention, les mérites des héros de la peinture sacrée. On peut se reporter par exemple à l'Ecce Homo qui se trouve à Bologne, dans l'église de Santa Maria del Borgo : les têtes des personnages qui, dans la partie inférieure du tableau, représentent le peuple hébreu à qui l'on montre le Christ, ont une allure bestiale avec leur museau féroce qui rappelle deux dessins encore inédits de têtes humaines avec une bouche, un nez et des oreilles de chien. Passarotti, qui s'essayait au grotesque avec un étrange acharnement, l'introduisait régulièrement dans la peinture sacrée, au risque d'encourir les foudres de Paleotti. L'évêque avait en effet consacré un des chapitres de son Discours aux "peintures ridicules" dans lequel il rappelait que le Concile de Trente les avait condamnées catégoriquement. Tous les tableaux d'autel de Passarotti, qui sont compris entre les deux dates que mentionne Malvasia, ont été composés entre 1570 et 1580, au moment où la campagne de Paleotti contre les abus de la peinture sacrée battait son plein. Passarotti ne s'en est donc pas soucié. Il a continué sur la voie qu'il avait choisie et que la tradition maniériste accréditait: il a adopté à l'égard de la peinture dévote une attitude de supériorité légèrement moqueuse. On sait pourtant qu'à la fin de cette décennie il a changé brusquement de registre: il a évité le nu, repoussé le grotesque, il s'est fait prudent et a cessé de se compromettre dans le domaine de la peinture sacrée, qu'il commençait de toute évidence à trouver dangereux. Il serait faux de croire qu'il s'est converti, comme Fontana et Cesi. En effet, contrairement à ces deux peintres, il n'a pas renoncé à cultiver ce qui l'intéressait dans la peinture profane; quant à la peinture sacrée, il a toujours maintenu ses distances par rapport aux courants dominants, comme en témoigne la Présentation de Marie au temple que mentionne Malvasia. On ne trouve, 
certes, aucune trace de nu ni de grotesque dans ce tableau, mais la construction d'ensemble, les gestes, la place des personnages et la distribution des parties, tout relègue, comme on l'a déjà remarqué, au second plan la Vierge Marie à qui pourtant ce tableau est consacré. Passarotti a donc feint de se convertir. Il a cherché à sauver les apparences et à faire croire, au moins dans ses tableaux d'église, qu'il suivait l'évêque dans le nouveau cours qu'à Bologne il avait imprimé à la peinture. On ne sait pas et, dans l'état actuel des recherches, il est impossible de savoir ce qui a amené le peintre à ce retournement. Ce qu'il y a de certain, c'est qu'en 1577 il a assumé pour la dernière fois la charge de massaro, et que jusqu'alors il avait entretenu d'assez bons rapports avec Fontana pour que celui-ci copie la Joyeuse compagnie; il s'agit là d'un des tableaux les plus bizarres de Passarotti, que l'on peut sans hésiter ranger parmi les peintures lascives si durement condamnées par Paleotti. Il a démissionné peu après du Conseil, juste au moment où commençaient les travaux pour les fresques du dôme. Il se peut que la méfiance qu'éprouvait certainement l'évêque à l'égard de ce peintre, qui ne tenait pas compte de ses recommandations, ait mis Passarotti en difficulté dans le Conseil. Mais il n'est pas exclu que sa démission soit liée à des remous qu'aurait provoquée son Adoration des Mages qui, d'abord exposée à San Pietro, a été transportée ailleurs, pour finalement retourner en plein $\mathrm{xvII}^{\mathrm{e}}$ siècle à son église d'origine. Ce tableau était en total désaccord avec les directives du Discours de Paleotti et il est possible, sans qu'on puisse pour l'instant le prouver, que l'évêque l'ait fait enlever. Quoiqu'il en soit, il est évident que Passarotti ne devait pas se sentir dans son élément à l'intérieur du Conseil, et qu'il a préféré démissionner 8

Pendant ce temps une nouvelle classe de jeunes s'apprêtait à prendre la relève. Parmi cette nouvelle génération se trouvaient des peintres qui allaient diriger la corporation au cours des vingt années suivantes. Le 28 juin 1582, Ludovic Carrache fut choisi par cooptation pour entrer dans le Conseil. Le 27 juin 1583, ce fut le tour de Bartolomeo Cesi. Ludovic avait été l'élève de Fontana, qui ne croyait pas à son talent et ne craignait donc nullement sa concurrence. Il entra dans le Conseil en même temps que Pietro Agnesina et Ercole Luchini, deux peintres absolument inoffensifs et inconnus dans les milieux artistiques de Bologne. Il en alla tout autrement avec Cesi qui, dès 1580, avait la réputation d'un peintre particulièrement prometteur. Fontana en avait conçu quelque inquiétude, et ne le fit admettre dans le Conseil qu'après qu'il se fût assuré que Cesi adhérait pleinement à la ligne de Palcotti. La bénédiction de l'évêque était certainement le meilleur viatique pour accéder à cette assemblée. Cesi s'y acquit très rapidement la confiance de tous les notables puisque dès 1586 il fut élu massaro. Il deviendra, avec le temps, à la suite de Fontana, le champion bolonais de la peinture dévote et donc le peintre préféré de Palcotti. Baglione entra finalement au Conseil, en même temps que Cesi, mais il dut au préalable accepter de participer aux fresques du dôme sur lesquelles Paleotti avait la haute main. Ce n'était pas là une acquisition de grand prix pour le Conseil, et c'est peut-être précisément cela qui décida de son choix. Baglione était souvent absent de Bologne; il travaillait dans la région de Parme, où il jouissait de la protection des Farnèse. Il pouvait y peindre en toute tranquillité les grotesques qu'il affectionnait, et contre lesquels Paleotti s'était si violemment insurgé dans son Discours qu'il n'y avait plus personne à Bologne pour se risquer encore dans ce genre.

En 1582, quand Ludovic, le plus âgé des trois Carrache, fut admis dans le Conseil, l'atelier qu'il avait ouvert peu avant à Bologne avec ses deux cousins Augustin et Annibal ne suscitait aucune inquiétude. Les trois peintres paraissaient adhérer parfaitement aux 
prescriptions de Paleotti et n'avoir nulle intention de semer le trouble dans la peinture locale. Moins d'un an après, Annibal, le plus jeune des trois, apportait à cette opinion un démenti cuisant : en 1583, âgé de vingt trois ans à peine, il jeta le premier caillou dans la mare avec sa célèbre Crucifixion. Ce n'était qu'une timide tentative, mais elle suffit à déclencher chez ses collègues plus âgés une réaction d'une violence extrême: ils lancèrent contre lui des attaques qui, comme d'habitude, mêlaient aux reproches techniques des accusations morales très clairement inspirées de Paleotti. Annibal dut donc mener son entreprise de rénovation de la peinture sous une pluie de critiques et sous la menace permanente de la censure ecclésiastique ${ }^{9}$.

\section{NOTES}

1. Sigles utilisés dans les notes: A.A.B., Archives de l'Archevêché de Bologne; A.S.B., Archives d'État de Bologne; A.S.R., Archives d'État de Rome; B.C.B., Bibliothèque communale de Bologne. D'autres recherches ont été menées par G. CAMMAROTA, "Cronacce della Compagna dei pittori » in Dall'avanguardia dei Carraci al secolo barocco, Bologna 1580-1600, Bologna, 1988, pp. 53 et sq. Les statuts de la corporation des peintres approuvés en 1569 se trouvent in A.S.B., Capitano del Popolo, Sociéta d'arti, Busta IV. Bombasari. D'autres documents ont été publiés par F. Malaguzzi Valeri, «L'arte dei pittori a Bologna nel secolo XVI », in Archivio storico dell'arte, serie II, III (1897), pp. 309 sq.

2. Pour les années les plus proches de celles qui nous intéressent, deux avis seulement ont été retrouvés : l'un du vice-légat Camillo Borghese du 30 janvier 1590, in B.C.B., Raccolta Merlani, II, c. $188 \mathrm{r}$, l'autre de son successeur Lorenzo Celsi, non daté, mais qui se situe entre mai 1592 et mars 1593, in A.S.B., Assunteria d'arti. Notizie sopra le arti. Bombasari e pittori. Pour le litige avec les dépendants de 1586-87 cf. A.S.B., Torrone, Registri di atti processuali, n. 1863, cc. 11r-13v; Assunteria d'arti. Notizie sopra le arti. Pittori. Memoriali. 1587-1696; Senato. Strumenti e scritture. 28.B.Lib.28, n. 34. La vente aux enchères des impôts des dépendants avait, pour les artisans les plus pauvres des conséquences dramatiques; quand ils étaient dans l'incapacité de payer, ils devaient se soumettre en échange aux travaux forcés. Voir sur ce point le travail de L. GHEZA FABBRI, «Le adunate segrete dei carpentieri bolognesi (1573-74)", in Atti dell'Academia delle scienze dell'Instituto di Bologna. Classe di scienze morali. Rendiconti. LVII (1978-79), fasc. II, pp. 25 sq. ; " Lavoro coatto e lavoro obbligato all'origine dell'età moderna : il caso di Bologna nel '500 e '600 », in Economia e storia, s. II, III (1982), pp. 323 sq. ; « Per uno studio del lavoro coatto in età moderna. Il caso di Bologna ", in Studi in memoria di Luigi Dal Pane, Bologna, 1982, pp. 427 sq.

3. Pour l'histoire de Desegna et Pinarizzi le 23 août 1577, A.S.B., Senato, Libri partitorum, Libro 10, c. 47 v. et C.C. MALVASIA, Felsina pittrice, ed. G. ZANOTTI, I, Bologna, 1842, pp. 169 sq., 258. Desegna était un pauvre diable; en 1583 il a reçu 25 écus du pape « en aumône pour que sa fille puisse devenir none ». On trouve la notification du payement dans A.S.R., Camerale I, Tesoreria segreta, vol. 1310, c. 15 v. Le jugement réservé de Malvasia sur Baglione se trouve dans Scritti originali del conte Carlo Cesare Malvasia spettanti alla sua Felsina pittrice, ed. L. MARZOCCHI, Bologna, s.d., pp. 120 sq. Il faut préciser pourtant que la critique moderne ne partage pas l'opinion de Malvasia: qu'on se reporte à la bibliographie citée par S. ZAMBONI in Dizionario biografico degli italiani, V, Roma, 1963, pp. 186 s., à G. Cirillo-G. GODI, « Di Orazio Samacchini e altri bolognesi a Parma », in Parma nell'arte, 14 (1982), pp. 7 sq. et à J. KLIEMANN, «Prospero Fontana in palazzo 
Vitelli a S. Edigio", in Mitteilungen des Kunsthistorischen Institutes in Florenz, XXXI (1987), pp. 186 sq. La Felsina pittrice reste un document indispensable. Malvasia lui consacre (I, pp. 253 sq.) l'une des biographies les plus savoureuses qu'il ait jamais écrites.

4. La liste du $1^{\mathrm{er}}$ octobre 1581 est publiée par Malaguzzi VALERI. p. 311. L'arrêté du Sénat du 31 janvier 1584 in A.S.B, Senato, Libri partitorum, Libro 11, c. 35 v. Au sujet de Zagnoni et Spinelli, voir MALVASIA, I, p. 277 ; II, p. 120.

5. Pour l'histoire de la composition et de la diffusion du Discours, voir P. PRODI, Ricerca sulla teoria delle arti figurative nella riforma cattolica. Bologna, 1984, pp. 30 sq. Les actes de ces visites pastorales se trouvent in A.A.A, Visite pastorali. 114. La condamnation des peintures lascives et les réflexions sur le rôle de la corporation dans le contrôle des arts visuels se trouvent dans Episcopale Bononiensis civitatis et diocesis..., Bologna. 1580, cc. 30 v., 37 v.. 216 v. G. PALEOTTI, «Discorso intorno al le immagini sacre et profane ", in Trattati d'arte del Cinquecento, a cura di P. BAROCCHI, II, Bari, 1962, pp. 496 sq.

6. A. BATTISTELLA, Il Sant'Offizio e la riforma religiosa in Bologna, Bologna, 1905, pp. 97, 99, 105 ; G. FOGOLARI, «Il processo dell'Inquisizione a Paolo Veronese », in Archivio veneto, LXV (1935), pp. 352 ss.

7. Pour tout ce qui concerne le sujet fort complexe de la censure du tableau de Federico Zuccari à Bologne, qu'on me permette de renvoyer à un article que j'ai consacré à cette question et qui doit être publié prochainement.

8. V. Fortunati PIETRANTONIO, «Cronaca di un "viaggio" attraverso la pittura bolognese nella Bologna pontificia del Cinquecento ", in Pittura bolognese del '500, I, Bologna, 1986, p. XXXVI s. fait explicitement référence à la résistance de Passarotti contre la pénétration subreptice de l'influence de l'évêque. Sur la question du nu, MALVASIA, I, pp. 187 s. Pour la chronologie des tableaux d'autel. A. GHIRARDI, «Bartolomeo Passarotti », in Pittura Bolognese del '500, II, cit., pp. 543 sq. ; pour le rapport avec le texte de Paleotti, «Discorso intorno alle immagini sacre e profane ", in Trattati d'arte del cinquecento, cit., II, pp. 391 sq., 502 sq. Les deux dessins inédits se trouvaient dans la galerie de Bernard Houthakker d'Amsterdam; j'ai pu en consulter les photographies à Paris à la photothèque du Cabinet des dessins du Louvre. Pour l'Adoration des Mages, voir la fiche de A. GHIRARDI in Bastiatino e la pittura a Ferrara nel secondo Cinquecento, Bologna, 1985, pp. 65 sq.

9. Sur toutes ces questions cf. R. ZAPPERI. Annibale Carrache.... op. cit.

\section{AUTEUR}

\section{ROBERTO ZAPPERI}

Roberto ZAPPERI est conseiller scientifique du Dizionario biografico degli Italiani. Ce texte a été présenté en décembre 1989 dans le cadre du séminaire de M. Jacques Revel. Voir également : R. ZAPPERI. Annibale Carrache. Portrait de l'artiste en jeune homme, Paris, Alinéa, 1990. 